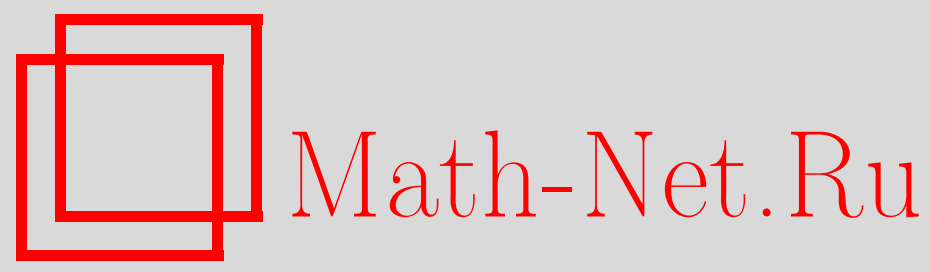

Г. А. Амбарцумян, А. В. Буробин, Продолжение функций, представляемых экспонентами бесконечной кратности с чередующимися показателями, Матем. заметки, 2003, том 73, выпуск 2, 163-172

DOI: https://doi.org/10.4213/mzm174

Использование Общероссийского математического портала Math-Net.Ru подразумевает, что вы прочитали и согласны с пользовательским соглашением http://www . mathnet.ru/rus/agreement

Параметры загрузки:

IP : 54.197 .217 .227

26 апреля 2023 г., 15:18:53

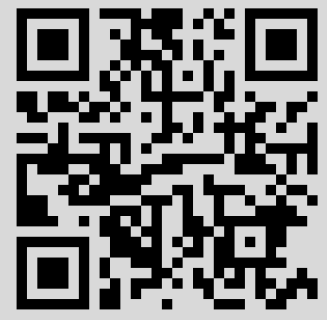


тОм 73 выпУСК 2 фЕВРАль 2003

\title{
ПРОДОЛЖЕНИЕ ФУНКЦИЙ, ПРЕДСТАВЛЯЕМЫХ ЭКСПОНЕНТАМИ БЕСКОНЕЧНОЙ КРАТНОСТИ \\ С ЧЕРЕДУЮШИМИСЯ ПОКАЗАТЕЛЯМИ
}

\section{Г. А. Амбарцумян, А. В. Буробин}

\begin{abstract}
Для экспонент бесконечной кратности с двумя чередующимися показателями строится продолжение на неограниченную область, являющееся предельной функцией последовательности экспонент конечной кратности. Доказывается, что при различных показателях такое продолжение единственно в классе непрерьвно дифференцируемых функций. Расширяется область существования экспонент бесконечной кратности. В случае показателей с одинаковьми знаками она остается ограниченной, а при показателях с разньпи знаками совпадает с числовой прямой.

Библиографиия: 7 названий.
\end{abstract}

Пусть задана числовая последовательность $\left\{a_{k}\right\}_{k=0}^{\infty}$, причем $a_{k} \neq 0, k=0,1, \ldots$ Следуя работе [1], будем назьвать гладкую функцию

$$
v_{n}(t)=a_{0} e^{a_{1} t e^{a_{2} t e^{\cdot} \cdot a_{n} t}} \quad, \quad n=0,1, \ldots,
$$

конечной кратной экспонентой, или әкспонентой кратности $n$, а поточечньй предел

$$
v_{\infty}(t)=\lim _{n \rightarrow \infty} v_{n}(t)=a_{0} e^{a_{1} t e^{a_{2} t e^{\cdot}}}
$$

последовательности экспонент конечной кратности

$$
\left\{v_{k}(t)\right\}_{k=0}^{\infty}
$$

- экспонентой бесконечной кратности.

Заметим, что с помощью простой замены переменной $z=e^{t}$ можно получить эквивалентное представление для экспонент (1) и (2) в виде степеней конечной и бесконечной кратности соответственно.

Экспонентам бесконечной кратности посвящено огромное количество работ, достаточно подробный обзор которых можно найти в работах [2] и [1]. В частности, одна из задач, во многом определивших первоначальный интерес к этим экспонентам, была 
посвящена изучению уравнения $x^{y}=y^{x}$, для решения которого достаточно найти обратную функцию для $z=g(x)=x^{1 / x}$, представимую степенью бесконечной кратности

$$
x=h(z)=z^{z^{z^{\circ}}} .
$$

Область существования этой степени была впервыеполучена Эйлером в 1778 году. Установлено, что $h(z)$ сушествует при $e^{-e} \leqslant z \leqslant e^{1 / e}$ и не существует при любом другом $z>0$.

Несмотря на то, что экспоненты бесконечной кратности практически полностью изучены для стационарных последовательностей $\left\{a_{k}\right\}_{k=0}^{\infty}$, сравнительно мало что известно про экспоненты с различными показателями. Одной из немногих фундаментальных работ, посвященных степеням бесконечной кратности с различными показателями, является работа Буланова [1]. В ней доказьвается, что степень бесконечной кратности при комплексной переменной $t$ и комплексных показателях $a_{k}$ является регулярной функцией в некоторой области на комплексной плоскости. В случае, когда переменная $t$ и показатели $a_{k}$ являются вещественньми, из доказанной теоремы следует, что экспонента бесконечной кратности (2) существует и является регулярной функцией на интервале $(-L, L)$, где

$$
L=\frac{1}{e \bar{a}}, \quad \bar{a}=\varlimsup_{n \rightarrow \infty}\left|a_{n}\right| .
$$

Однако областью сходимости, полученной Эйлером для экспоненты бесконечной кратности

$$
v_{\infty}(t)=e^{t e^{t e^{\cdot}}}
$$

является отрезок $\left[-e, e^{-1}\right]$, правильной частью которого является интервал $(-L, L)$. Возникают естественные вопросы о возможности расширения области существования экспоненты (2) и продолжения функции, задаваемой этой экспонентой, за пределы интервала. Из работы [3] известно, что для чередующихся показателей $a_{2 k}=\alpha$, $a_{2 k+1}=\beta, k=0,1, \ldots$, область существования экспоненты бесконечной кратности

$$
v_{\infty}(t)=\alpha e^{\beta t e^{\alpha t e^{\beta t e^{\cdot \cdot}}}}
$$

может быть расширена следующим образом. Пусть $|\alpha|=1,|\beta|>1$. Тогда вместо $\bar{a}=|\beta|$ в определении величины $L$ можно взять

$$
\bar{a}=\sqrt{|\beta|}\left(1+\frac{1}{2} \ln |\beta| \frac{\sqrt{|\beta|}-1}{\sqrt{|\beta|}+1}\right)
$$

Но, очевидно, полученная таким образом область существования остается конечным интервалом.

В данной работе доказывается возможность продолжения функции, задаваемой экспонентой (4), на неограниченную область, причем это продолжение является предельной функцией последовательности экспонент конечной кратности (3). Вид этой области зависит от алгебраических знаков $\alpha$ и $\beta$. Существенными оказьваются два случая: 
$\alpha \beta>0$ и $\alpha \beta<0$. Первьй случай был частично исследован авторами в работе [4], в которой рассматривались положительные показатели $\alpha$ и $\beta$. Легко заметить, что случай отрицательных $\alpha$ и $\beta$ сводится к предыдущему изменением знака переменной $t$.

Пусть $\alpha>0, \beta>0$. Нобель [2] доказал, что если $\alpha=\beta=1$, то последовательность экспонент конечной кратности (3) монотонно и неограниченно возрастает при $t>e^{-1}$ и имеет частичные пределы, осциллируя в ограниченной области, при $t<-e$. Поэтому естественным является поиск продолжения функции, задаваемой экспонентой (4), на область $\{t: t<L\}$.

Tеорема 1. Пусть $\alpha>0, \beta>0 u \alpha \neq \beta$. Тогда функиия $v_{\infty}(t)$ имеет единственное продолжение, являющееся предельной функиией последовательности әкспонент конечной кратности (3), в классе непрерывно дифференцируемых функций на множество $\{t: t<L\}$.

ДокАЗАТЕЛЬСтво. Существование экспоненты бесконечной кратности на интервале $(-L, L)$ следует из работы Буланова [1]. Поэтому в дальнейшем будем считать, что $t<0$.

Установим ряд вспомогательных фактов, справедливых при произвольных положительных показателях $\alpha$ и $\beta$.

Для экспонент конечной кратности имеют место соотношения

$$
v_{2 k+1}(t)=\alpha e^{\beta t e^{v_{2 k-1}(t) t}}, \quad v_{2 k}(t)=\alpha e^{\beta t e^{v_{2 k-2}(t) t}}, \quad k=1,2, \ldots
$$

предельный переход в которых показьвает, что в области своего существования экспонента (4) удовлетворяет конечному уравнению

$$
v(t)=\alpha e^{\beta t e^{v(t) t}} .
$$

Заметим, однако, что, вообще говоря, не все решения этого конечного уравнения удается получить с помощью экспоненты (4).

Последовательность экспонент конечной кратности с нечетными номерами

$$
\left\{v_{2 k+1}(t)\right\}_{k=0}^{\infty}
$$

является монотонно возрастающей и ограничена сверху функцией $v(t) \equiv \alpha$, а последовательность экспонент конечной кратности с четными номерами

$$
\left\{v_{2 k}(t)\right\}_{k=0}^{\infty}
$$

монотонно убывает и ограничена снизу функцией $v(t) \equiv 0$. Действительно, отображение $E:[0, \alpha] \rightarrow[0, \alpha]$,

$$
g=E(f)=\alpha e^{\beta t e^{f t}}
$$

при фиксированном $t$ является монотонно возрастаюшим, и имеют место рекуррентные соотношения

$$
v_{2 k+1}=E\left(v_{2 k-1}\right), \quad v_{2 k}=E\left(v_{2 k-2}\right), \quad k=1,2, \ldots,
$$


причем $v_{2}(t)<v_{0}(t)$ и $v_{1}(t)<v_{3}(t)$. Следовательно, последовательности (6) и (7) поточечно сходятся, а их пределы представляют собой полунепрерьвную снизу и полунепрерьвную сверху функции соответственно (см. [5]), которые удовлетворяют уравнению (5). Понятно, что при $t \in(-L, 0)$ они совпадают и являются непрерывными на этом интервале.

Рассмотрим функцию

$$
F(t, v)=v-\alpha e^{\beta t e^{v t}}
$$

Обозначим через $Z(F)$ множество точек $(t, v)$, удовлетворяющих уравнению (5). Частные производные функции $F(t, v)$ непрерывны на всей плоскости $\mathbb{R}^{2}$ и на множестве $Z(F)$ имеют вид

$$
F_{v}^{\prime}(t, v)=1-\beta v t^{2} e^{v t}, \quad F_{t}^{\prime}(t, v)=-\beta v(1+v t) e^{v t} .
$$

Из работы [6] известно, что при $t \in(-L, L)$ функция $v_{\infty}(t)$ является решением задачи Коши

$$
\begin{aligned}
& v^{\prime}=\beta v e^{v t} \frac{1+v t}{1-\beta v t^{2} e^{v t}}, \\
& v(0)=\alpha .
\end{aligned}
$$

Можно легко показать, что функция $v_{\infty}(t)$ является решением задачи Коши для дифференциального уравнения (9) с начальным условием более общего вида:

$$
v\left(t_{0}\right)=v_{0}
$$

если точка $\left(t_{0}, v_{0}\right) \in Z(F)$ и не является решением уравнения

$$
D(t, v)=1-\beta v t^{2} e^{v t}=0 .
$$

Такие точки будем назьвать регулярными для $Z(F)$ (см. [7]). Примером регулярной точки является точка $(0, \alpha)$. Продолжение функции $v_{\infty}(t)$ получается при продолжении решения этой задачи Коши.

Лемма 1. Пусть $\left(t_{0}, v_{0}\right)$ - регулярная точка. Тогда в некоторой окрестности этой точки задача Коши (9), (11) и конечное уравнение (5) однозначно разрешимы $u$ определяют одно и то же решение $v(t)$.

ДокАЗАТЕЛЬСТВО. В окрестности регулярной точки $\left(t_{0}, v_{0}\right)$ правая часть уравнения (9) является непрерывно дифференцируемой функцией и, следовательно, решение задачи Коши $(9),(11)$ единственно в этой окрестности. Оно удовлетворяет уравнению

$$
v(t)=v_{0} e^{-\beta t_{0} e^{v_{0} t_{0}}} e^{\beta t e^{v(t) t}},
$$

которое совпадает с уравнением (5), так как для регулярной точки $\left(t_{0}, v_{0}\right)$ вьполняется условие

$$
v_{0}=\alpha e^{\beta t_{0} e^{v_{0} t_{0}}} .
$$


Из справедливости соотношений $F\left(t_{0}, v_{0}\right)=0, F_{v}^{\prime}\left(t_{0}, v_{0}\right) \neq 0$ следует, что в некоторой окрестности точки $\left(t_{0}, v_{0}\right)$ уравнение $(5)$ определяет $v(t)$ как однозначную и непрерьвно дифференцируемую функцию переменной $t$, которая является решением задачи Коши (9), (11). Лемма доказана.

Исследуем множество критических (нерегулярных) точек $(t, v) \in Z(F)$. Будем называть точкой поворота множества $Z(F)$ (см. [7]) любую критическую точку $\left(t_{p}, v_{p}\right)$, в которой

$$
F_{t}^{\prime}\left(t_{p}, v_{p}\right) \neq 0
$$

а производная функции $t(v)$, задаваемой уравнением (5) в окрестности этой точки, меняет знак. При переходе $t$ через значение $t_{p}$ происходит бифуркация: появляется или исчезает пара решений $v(t)$ уравнения (5). Все остальные критические точки будем назьвать сингулярными (см. [7]). Обозначим через $P(F)$ множество точек $(t, v)$, удовлетворяюших конечному уравнению (12).

Лемма 2. Множество критических точек $Z(F)$ совпадает с множеством $Z(F) \cap P(F)$. При $\alpha=\beta$ оно содержит единственную сингуляную точку $S=$ $(-e / \beta, \beta / e)$. При $\alpha \neq \beta$ сингулярных точек нет.

ДокАЗАТЕЛЬСТВо. Из условия (14) следует, что $\left(t_{p}, v_{p}\right)$ не является решением уравнения

$$
1+v t=0
$$

Система уравнений (12) и (15) имеет только одно общее решение $S=(-e / \beta, \beta / e)$. Подставляя его в конечное уравнение $(5)$, получаем, что $\beta / \alpha=1$, т.е. сингулярная точка $S$ принадлежит множеству $Z(F)$ только в случае $\alpha=\beta$. Если $Z(F) \cap P(F) \backslash\{S\} \neq \varnothing$, то из отрищательности $t^{\prime \prime}(v)$ в любой точке $\left(t_{1}, v_{1}\right) \in Z(F) \cap P(F) \backslash\{S\}$ следует, что $\left(t_{1}, v_{1}\right)$ является точкой локального максимума для функции $t(v)$ и точкой поворота для множества $Z(F)$. Лемма доказана.

Рассмотрим подробнее множество $P(F)$. Частные производные функции $D(t, v)$ имеют вид

$$
D_{v}^{\prime}(t, v)=-\beta t^{2} e^{v t}(1+v t), \quad D_{t}^{\prime}(t, v)=-\beta v t e^{v t}(2+v t) .
$$

Точки из $P(F)$, в которых $D_{v}^{\prime} \neq 0$, т.е. не вьполняется равенство $(15)$, являются регулярными для $P(F)$. В окрестности любой такой точки уравнение (12) определяет непрерьвно дифференцируемую функцию $v=v(t)$. Единственной точкой из $P(F)$, не удовлетворяющей этому условию, является точка $S$. Она является точкой поворота для $P(F)$ и точкой локального максимума для функции $t(v)$, задаваемой уравнением (12) в окрестности $S$, так как справедливы соотношения $\left.D_{t}^{\prime}(t, v)\right|_{S} \neq 0,\left.t^{\prime \prime}(v)\right|_{S}<0$. Функция $v(t)$ имеет точку глобального максимума $t_{m}=e^{2} /(2 \beta)$, в которой достигает значения $v_{m}=4 \beta e^{-2}$, и других точек экстремума она не имеет.

Для дальнейшего доказательства важной является геометрическая структура множеств $Z(F)$ и $P(F)$. Критические точки разбивают эти множества на ветви решений уравнений (5) и (12) соответсвенно (см. [7]). Каждая ветвь состоит из регулярных точек и определяет однозначную гладкую зависимость $v(t)$. Иначе говоря, ветви - компоненты связности множества регулярных точек. Если решение уравнения на какой-либо области состоит из двух непересекающихся ветвей, то будем называть их в соответствии с расположением на плоскости верхней и нижней ветвями. 
Изучим поведение линии, состоящей из точек множества $P(F)$, при $t \rightarrow-\infty$. Введем параметр $w=v t<0$. Тогда уравнение (12) приводится к виду $1-\beta t w e^{w}=0$, а линия $P(F)$ задается параметрически: $t=\left(\beta w e^{w}\right)^{-1}, v=\beta w^{2} e^{w}$. Очевидно, что $t \rightarrow-\infty$, если $w \rightarrow-0$ либо $w \rightarrow-\infty$. В обоих случаях $v \rightarrow+0$.

Таким образом, мы показали, что линия $P(F)$ состоит из двух непересекающихся ветвей, определяющих гладкую зависимость $v(t)$ на полуоси $(-\infty,-e / \beta)$ и точки поворота $S$. Функция $v(t)$ достигает глобального максимума на верхней ветви и монотонно возрастает на нижней. При этом на обеих ветвях $v(t) \rightarrow 0$ при $t \rightarrow-\infty$.

Полученное означает, что при $t \in(-e / \beta, 0)$ множество $Z(F)$ не имеет критических точек; следовательно, уравнение (5) определяет $v(t)$ на этом интервале как однозначную непрерьвно дифференцируемую функцию.

Как ранее было отмечено, гипербола (15) пересекает $P(F)$ только в точке $S$, где имеет конечный положительный угловой коэффициент. Отсюда следует, что эта гипербола находится между верхней и нижней ветвями $P(F)$ при $t<-e / \beta$. Из дифференциального уравнения (9) видно, что гипербола (15) и линия $P(F)$ разбивают полуполосу $\{(t, v):-\infty<t<0,0<v<\alpha\}$ на четыре области монотонности функции $v(t)$, удовлетворяющей уравнению (5):

$$
\begin{aligned}
& d_{1}=\left\{(t, v): 1+v t>0,1-\beta v t^{2} e^{v t}>0\right\}, \\
& d_{2}=\left\{(t, v): 1+v t<0,1-\beta v t^{2} e^{v t}>0\right\}, \\
& d_{3}=\left\{(t, v): 1+v t<0,1-\beta v t^{2} e^{v t}<0\right\}, \\
& d_{4}=\left\{(t, v): 1+v t>0,1-\beta v t^{2} e^{v t}<0\right\} .
\end{aligned}
$$

В областях $d_{1}$ и $d_{3}$ функция $v(t)$ монотонно возрастает, а в областях $d_{2}$ и $d_{4}$ монотонно убывает.

Исследуем множество $Z(F)$ при $\alpha=\beta$ в окрестности сингулярной точки и за ее пределами.

Лемма 3. Пусть $\alpha=\beta$. Тогда множество точек $(t, v) \in Z(F)$ состоит из одной ветви при $t \in(-e / \beta, 0)$ и трех непересекающихся ветвей, выходящих из точки $S=(-e / \beta, \beta / e)$, при $t \in(-\infty,-e / \beta)$.

ДоказАТЕЛЬСтво. Разложим функцию $F(t, v)$ по формуле Тейлора в окрестности точки $S=\left(t_{s}, v_{s}\right)=(-e / \beta, \beta / e)$. Тогда уравнение $(5)$ перепишется в виде

$$
\frac{1}{2}\left[A\left(v-v_{s}\right)^{2}+2 B\left(v-v_{s}\right)\left(t-t_{s}\right)+C\left(t-t_{s}\right)^{2}\right]+o\left[\left(v-v_{s}\right)^{2}+\left(t-t_{s}\right)^{2}\right]=0,
$$

где $A=F_{v v}^{\prime \prime}\left(t_{s}, v_{s}\right)=0, B=F_{v t}^{\prime \prime}\left(t_{s}, v_{s}\right)=F_{t v}^{\prime \prime}\left(t_{s}, v_{s}\right)=\beta / e, C=F_{t t}^{\prime \prime}\left(t_{s}, v_{s}\right)=-\beta^{3} e^{-3}$. Пусть $v \neq v_{s}$. Разделим обе части этого уравнения на $\left(v-v_{s}\right)^{2}$ и осуществим предельньй переход $(t, v) \rightarrow\left(t_{s}, v_{s}\right)$ при $(t, v) \in Z(F)$. Получим уравнение

$$
C\left(\frac{d t}{d v}\right)^{2}+2 B \frac{d t}{d v}+A=0
$$

из которого можно заключить, что либо $t^{\prime}(v)=0$, либо $t^{\prime}(v)=2 e^{2} \beta^{-2}$. Отсюда следует, что множество $Z(F)$ в окрестности сингулярной точки $S$ состоит из двух пересекающихся дуг. Одна из них имеет в этой точке вертикальную касательную, так что точка $S$ 
является точкой поворота для этой дуги (точкой максимума для $t(v)$ ), а вторая имеет касательную с угловым коэффициентом $k=2 e^{2} \beta^{-2}$ и при $t>-e / \beta$ совпадает с графиком единственного решения $v(t)$ уравнения (5). В точке $S$ имеет место бифуркация типа "вилка" (см. [7]). Из свойств полученных дуг следует, что существует $\varepsilon>0$ такое, что при $t \in(-e / \beta-\varepsilon,-e / \beta)$ множество $Z(F)$ состоит из трех непересекающихся ветвей, выходящих из точки $S$, причем верхняя и нижняя ветви имеют гладкое соединение в точке $S$ с вертикальной касательной и находятся в областях $d_{2}$ и $d_{1}$ соответственно, а средняя находится в области $d_{3}$.

Рассмотрим задачу Коши $(9),(11)$ с начальной точкой $\left(t_{0}, v_{0}\right)$ на верхней ветви множества $Z(F)$. По лемме 1 решение $v(t)$ этой задачи единственно в некоторой окрестности точки $t_{0} \in(-e / \beta-\varepsilon,-e / \beta)$ и удовлетворяет уравнению (5). При продолжении влево ее график не пересечет линию $P(F)$. Докажем последнее утверждение.

Пусть график продолжения решения пересекает $P(F)$. По лемме 2 общая для $Z(F)$ и $P(F) \backslash\{S\}$ точка является точкой поворота. Но для любой точки поворота $\left(t_{p}, v_{p}\right)$ можно указать $\delta>0$ такое, что прямоугольник $\left\{(t, v): t_{p}<t<t_{p}+\delta, v_{p}-\delta<v<v_{p}+\delta\right\}$ не будет содержать точек из $Z(F)$. А это приводит к противоречию: в точку поворота $\left(t_{p}, v_{p}\right)$ график продолжения решения задачи Коши может попасть только через этот прямоугольник, что и завершает доказательство утверждения.

Из доказанного следует, что существует число $\delta_{1}>0$ такое, что $D(t, v)>\delta_{1}$ при $t \in\left(-\infty, t_{0}\right)$, а это значит, что правая часть уравнения (9) имеет ограниченную производную по $v$ на интервале $\left(-\infty, t_{0}\right)$ и, следовательно, решение задачи Коши можно продолжить на всю отрищательную полуось. Аналогично можно доказать, что средняя и нижняя ветви $Z(F)$ продолжимы на всю отрицательную полуось. Заметим также, что $Z(F)$ имеет только одну общую точку $S$ с гиперболой (15) и, значит, средняя ветвь $Z(F)$ всегда остается в области $d_{3}$. На средней и нижней ветвях $v(t) \rightarrow 0$ при $t \rightarrow-\infty$. Докажем, что на верхней ветви $v(t) \rightarrow \alpha$ при $t \rightarrow-\infty$.

Нижнюю и верхнюю ветви $Z(F)$ можно получить как пределы последовательностей (6) и (7) соответственно, так как $Z(F)$ является множеством неподвижных точек отображения (8). При $\alpha=\beta$ справедливы соотношения $v_{2 k}(t)=\alpha e^{v_{2 k-1}(t)}, k=1$, $2, \ldots$, а поскольку

$$
\lim _{t \rightarrow-\infty} \lim _{k \rightarrow \infty} v_{2 k-1}(t)=0
$$

TO

$$
\lim _{t \rightarrow-\infty} \lim _{k \rightarrow \infty} v_{2 k}(t)=\alpha
$$

Лемма доказана.

Вернемся к доказательству теоремы. Пусть $\alpha>\beta$. График решения задачи Коши $(9),(10)$ не имеет общих точек с множеством $P(F)$, так как он изначально расположен выше графика решения дифференциального уравнения (9) с вырожденным начальньп условием $v(0)=\alpha_{0}=\beta$ и не пересекает его ни в одной регулярной точке. $\mathrm{B}$ противном случае нарушилась бы единственность решения задачи Коши в точке пересечения. Заметим, что в силу леммы 2 решение задачи Коши $(9),(10)$ не может попасть в сингулярную точку $S$. Отделенность продолжения решения задачи Коши (9), (10) от множества $P(F)$ означает возможность этого продолжения на всю отрищательную полуось. Полученное продолжение удовлетворяет конечному уравнению (5) и является пределом последовательности (7). 
Аналогично рассматривается случай $\alpha<\beta$. Полученное при этом продолжение является пределом последовательности (6), что и завершает доказательство теоремы 1.

СлЕДСТВИЕ 1. Построенное продолжение функиии $v_{\infty}(t)$ является пределом последовательности (6) при $0<\alpha<\beta$ и последовательности (7) при $\alpha>\beta>0$, т.е. является частичным пределом последовательности әкспонент конечной кратности (3).

Из леммы 3 следует, что при $\alpha=\beta>0$ функция $v_{\infty}(t)$ не имеет гладкого продолжения, являющегося предельной функцией последовательности (3) экспонент конечной кратности, за пределы отрезка $\left[e / \beta,(e \beta)^{-1}\right]$. Однако существуют два различных непрерывных продолжения этой функции на множество $\left\{t: t<(e \beta)^{-1}\right\}$, одно из которых является пределом последовательности (6), а другое - пределом последовательности (7) экспонент конечной кратности. Это объясняет причину осциллящии последовательности (3) вне области сходимости, установленной Эйлером, при наличии частичных пределов.

Оказьвается, что и при $\alpha \neq \beta$ область существования экспоненты бесконечной кратности остается конечньм интервалом, хотя и существенно расширяется.

Tеорема 2. Пусть $\alpha>0, \beta>0 u \alpha \neq \beta$. Тогда существуют иисла $T>$ е $/ \beta u$ $V \in(0, \alpha)$ такие, что мнохество точек $(t, v) \in Z(F)$ состоит из одной ветви при $t \in(-T, 0)$ и трех непересекающихся ветвей, выходящих из точки $(-T, V)$, при $t \in(-\infty,-T)$.

ДокАЗАтЕЛЬСтво. Рассмотрим задачу Коши (9), (11) при условии, что точка $\left(t_{0}, v_{0}\right)$ является регулярной и лежит в области $o_{1}$ между средней и верхней или в области $o_{2}$ между средней и нижней ветвями решения уравнения (5) при $\alpha=\beta$. Ee решение удовлетворяет уравнению (13). Введем обозначение

$$
\alpha_{1}=v_{0} e^{-\beta t_{0} e^{v_{0} t_{0}}}
$$

Тогда уравнение (13) совпадет с уравнением (5) при $\alpha=\alpha_{1}$. Пользуясь такими же рассуждениями, как в доказательстве предыдущей теоремы, можно показать, что решение задачи Коши продолжается влево на область $\left(-\infty, t_{0}\right)$ и вправо до некоторой точки $(-T, V)$, которая является точкой поворота $Z(F)$ при $\alpha=\alpha_{1}$, единственньм образом. При этом $T=T\left(\alpha_{1}, \beta\right)>e / \beta, V=V\left(\alpha_{1}, \beta\right) \in\left(0, \alpha_{1}\right)$. В этой точке из $P(F)$ гладко соединяются две ветви решения уравнения (5). Вторая ветвь также существует на всей области $(-\infty,-T)$. Точка поворота однозначно определяет значение $\alpha_{1}$ для линии, состоящей из этих ветвей и образующей отдельную компоненту связности $Z(F)$. Другой компонентой связности является график продолжения решения задачи Коши (9), (10) на отрицательную полуось.

Непрерывно дифференцируемая функция $\alpha_{1}\left(v_{0}\right)$ достигает своего минимума на верхней ветви множества $P(F)$ и максимума - на нижней. Следовательно, $\alpha_{1}>\beta$ в области $o_{2}$ и $\alpha_{1}<\beta$ в области $o_{1}$.

Пусть $\alpha_{1}<\beta$. Поскольку графики решений задачи Коши (9), (11) при различных начальных условиях не могут пересекаться в регулярной точке, то из графиков двух решений с разными $\alpha_{1}$ точка поворота левее у того, у которого $\alpha_{1}$ меньше. Значение $\alpha_{1}$ в точке $\left(t_{0}, v_{0}\right)$ на $P(F)$ задается формулой $\alpha_{1}=v_{0} e^{-1 /\left(v_{0} t_{0}\right)}$. В предыдущей теореме 
мы доказали, что если на верхней ветви $P(F) t \rightarrow-\infty$, то $v \rightarrow+0$ и $v t \rightarrow-\infty$. Следовательно, $\alpha_{1} \rightarrow 0$ при $t \rightarrow-\infty$ на этой ветви, а это значит, что при фиксированном $\beta$ для любого $\alpha<\beta$ найдется точка $T(\alpha, \beta)>0$ такая, что на интервале $(-T, 0)$ множество $Z(F)$ состоит из одной ветви, а на интервале $(-\infty,-T)$ - из трех ветвей. Верхняя и средняя ветви вьходят из одной точки поворота $(-T, V)$. Нижняя ветвь является пределом последовательности (6), а верхняя - пределом последовательности (7).

В случае $\alpha_{1}>\beta$ доказательство сводится к предыдущему, так как с точки зрения сходимости последовательности (3) параметры $\alpha$ и $\beta$ эквивалентны. Теорема 2 доказана.

СлЕДСТВИЕ 2. При $\alpha>0, \beta>0$ область существования әкспоненты бесконечной кратности (4) ограничена.

СлЕДСТВИЕ 3. Для любых $\beta>0 u T>0$ можно подобрать $\alpha>0$ maкое, что әкспонента бесконечной кратности (4) будет существовать на интервале $(-T, L)$.

СЛЕДСТВИЕ 4. При $\alpha>0, \beta>0$ әкспонента бесконечной кратности (4) имеет бесконечно много разрывных продолжений, являющихся предельными функииями последовательности (3) әкспонент конечной кратности, на область $(-\infty, L)$.

В случае, когда параметры $\alpha$ и $\beta$ имеют разные знаки, удается доказать глобальное существование экспоненты бесконечной кратности.

Tеорема 3. Пусть $\alpha \beta<0$. Тогда әкспонента бесконечной кратности (4) существует на всей числовой оси и является непрерывно дифференцируемой ограниченной функиией.

ДокаЗАТЕЛьство. Исследуем решение задачи Коши (9), (10) при $\alpha>0, \beta<0$. Лемма 1 в этом случае остается в силе. Уравнение (12) не имеет решения. Решение $v(t)$ уравнения (5) ограничено константой $\alpha$ при $t>0$ и функцией $f=\alpha e^{\beta t}$ при $t<0$. Следовательно, существует единственное продолжение решения задачи Коши на всю числовую ось. Все точки из $Z(F)$ регулярны, и конечное уравнение $(5)$ определяет $v(t)$ как однозначную непрерывно дифференцируемую функцию на всей числовой прямой.

Отображение (8) является монотонно убывающим. К последовательности экспонент конечной кратности (3) добавим еще одну функцию $v_{-1} \equiv 0$. Заметим, что $v_{0}=E\left(v_{-1}\right)$. Тогда при любом $k=0,1, \ldots$ справедливы соотношения $v_{2 k-1}<v_{2 k+1}<v_{2 k+2}<v_{2 k}$, из которых следует, что последовательность (6) монотонно возрастает и ограничена сверху функцией $v_{0}(t)$, а последовательность (7) монотонно убьвает и ограничена снизу функцией $v_{1}(t)$. Отображение (8) при фиксированном $t$ имеет одну неподвижную точку. Следовательно, последовательности (6) и (7) сходятся к одной и той же функции, являющейся экспонентой бесконечной кратности, на всей числовой прямой. Точка пересечения гиперболы $(15)$ и графика экспоненты бесконечной кратности является точкой максимума для этой экспоненты.

Случай $\alpha<0, \beta>0$ рассматривается аналогично. Теорема 3 доказана.

Авторы выражают признательность А. П. Буланову за вызванный им интерес к данной задаче и за поддержку. 


\section{СПИСОК ЦИТИРОВАННОЙ ЛИТЕРАТУРЫ}

[1] Буланов А. П. Регулярность степеней бесконечной кратности // Изв. РАН. Сер. матем. 1998. Т. 62. № 5. C. 49-78.

[2] Knoebel A. Exponentials reiterated // Amer. Math. Monthly. 1981. V. 88. №4. P. 235-252.

[3] Буланов А. П. О степени бесконечной кратности с коэффициентами, имеющими поочередно два значения // Современные проблемы теории функций и их приложения. Тезисы докладов 9-й Саратовской зимней школы. Саратов: Изд-во Саратовского ун-та, 1998. С. 31.

[4] Амбарцумян Г. А., Буробин А. В. О продолжении функций, представляемых экспонентами бесконечной кратности // Современные методы теории функций и смежные проблемы. Тезисы докладов Воронежской зимней математической школы. Воронеж: ВГУ, 1999. С. 16.

[5] Натансон И. П. Теория функций вещественной переменной. М.: Наука, 1974.

[6] Буланов А. П. О сходимости кратной степени, являющейся решением нелинейного дифференциального уравнения // Алгебра и анализ. Материалы конференции, посвященной 100-летию Б. М. Гагаева (16-22 июня 1997 г., г. Казань). Казань: Изд-во Казанского математического общества, 1997. С. 40-41.

[7] Холодниок М., Клич А., Кубичек М., Марек М. Методы анализа нелинейных динамических моделей. М.: Мир, 1991.

Обнинский институт атомной энергетики

Поступило

E-mail: zokhaik@mail.ru, avbur@chat.ru 12.10 .2000 